\title{
Clinical effect of decitabine in the treatment of myelodysplastic syndrome and influencing factors
}

\author{
Tangxia Liu' ${ }^{1}$ Jing Wang ${ }^{2}$, \\ Chunmei Li ${ }^{3}$, Lingzhi Jia ${ }^{4}$
}

\begin{abstract}
Objective: To analyze the effect of decitabine combined with conventional chemotherapy on myelodysplastic syndrome (MDS) and its influencing factors.

Methods: Eighty patients with MDS who were admitted to our hospital were selected by this study between February 2017 and February 2018. The selected patients were divided into a traditional group (CAG/DA scheme) and the combined group (DAC combined with CAG/DA scheme) according to the random number table method, 40 each. The clinical treatment effects of the two groups were compared, and the influencing factors of the effect were analyzed.

Results: After four courses of treatment, the difference in the total effective rate between the two groups were statistically significant $(\mathrm{P}<0.05)$; the difference in the incidence of adverse reactions and in the overall survival (OS) rate between the two groups were not statistically significant $(P>0.05)$. However, the progression free survival (PFS) rate of the combined group was significantly higher compared with the traditional group $(\mathrm{P}<0.05)$; the $\mathrm{Hb}$ level, WHO stage and and karyotype of the patients before treatment had significant influence on the treatment effect $(P<0.05)$.

Conclusion: DAC in combination with conventional chemotherapy has good effect in the treatment of MDS, and it will not increase adverse reactions. In addition to treatment scheme, the influencing factors of the effect of treatment for MDS also include $\mathrm{Hb}$, WHO stage and karyotype.
\end{abstract}

KEYWORDS: Decitabine; Myelodysplastic syndrome; CAG; Scheme.

doi: https://doi.org/10.12669/pjms.36.5.2289

How to cite this:

Liu T, Wang J, Li C, Jia L. Clinical effect of decitabine in the treatment of myelodysplastic syndrome and influencing factors. Pak J Med Sci. 2020;36(5):1084-1088. doi: https://doi.org/10.12669/pjms.36.5.2289

This is an Open Access article distributed under the terms of the Creative Commons Attribution License (http://creativecommons.org/licenses/by/3.0), which permits unrestricted use, distribution, and reproduction in any medium, provided the original work is properly cited.

1. Tangxia Liu,

Hematology Department,

2. Jing Wang,

Mental Health Center,

3. Chunmei Li,

Digestive Endoscopy Center,

4. Lingzhi Jia

Hematology Department,

Binzhou Medical University Hospital,

Shandong, 256603, China.

1-3: Binzhou People's Hospital,

Shandong, 256610, China.

Correspondence:

Lingzhi Jia

No. 661, Huanghe $2^{\text {nd }}$ Road,

Binzhou Medical University Hospital,

Shandong, 256603, China.

E-mail: jialingzhiy@163.com

* Received for Publication:

* Accepted for Publication:
February 11, 2020

May 30, 2020

\section{INTRODUCTION}

Myelodysplastic syndrome (MDS) is a common disease in the blood system, which is mainly caused by the abnormal development of myeloid cells and ineffective hematopoiesis. It is characterized by the increase of primordial cells and the increase and morphological abnormality of of bone marrow nucleated cells. ${ }^{1,2}$ Clinically, there is a high risk of MDS turning into acute myeloid leukemia, and the incidence of MDS tends to increase with age. ${ }^{3}$ At present, there are many treatment methods for MDS, but there is no unified method. In recent years, a relevant research shows that DNA methylation is a common phenomenon in MDS patients and the inactivation of tumor suppressor gene caused by hypermethylation is closely 
related to the progress of MDS. ${ }^{4}$ The diagnosis and treatment guidelines of European Leukemia Net recommend methyltransferase inhibitors for treatment of MDS. ${ }^{5}$ Decitabine is a specific DNA methyltransferase inhibitor, which can induce tumor cells to turn into normal cells or to die. ${ }^{6,7}$ However, the clinical application time of decitabine in China is relatively short, and large sample data are not yet available to support whether factors such as Revised International Prognostic Scoring System (IPSS-R) risk stratification and genetic difference will affect the treatment effect of DAC for MDS. Therefore, this study explored the effect of DAC combined with conventional chemotherapy on MDS and its influencing factors, in order to provide more optimal choices for clinical practice.

\section{METHODS}

Eighty MDS patients who were admitted to our hospital were selected as the study subjects from December 2017 to December 2018. All selected patients were divided into a traditional group (CAG/DA scheme) and a combined group (DAC combined CAG/DA scheme) according to the random number table method.

There were 40 patients in the traditional group, including 22 males and 19 females, and they aged $21-67$ years, with an average age of $(43.3 \pm 12.5)$ years. Before treatment, the level of peripheral white blood cells $(\mathrm{WBC})$ was $(17.5 \pm 5.3) \times 10^{9} / \mathrm{L}$, the level of platelets (PLT) was $(67.4 \pm 31.9) \times 10^{\circ} / \mathrm{L}$, and the level of hemoglobin $(\mathrm{Hb})$ was $(72.3 \pm 18.9)$ $\mathrm{g} / \mathrm{L}$. As to the WHO stage, there were 24 cases of RAEB-1 stage and 16 cases of RAEB-2 stage. As to the IPSS score, there were 0 case of low risk, 26 cases of medium risk and 14 cases of high risk. As to the karyotype, there were 21 good cases, 13 moderate cases and six bad cases. There were 40 patients in the combined group, including 29 males and 11 females, and they aged 19-61 years, with an average age of $(39.7 \pm 15.1)$ years. Before treatment, the level of WBC was $(18.2 \pm 5.6) \times 10^{9} / \mathrm{L}$, the level of PLT was $(66.1 \pm 28.6) \times 10^{9} / \mathrm{L}$, and the level of $\mathrm{Hb}$ was $(70.8 \pm 16.2) \mathrm{g} / \mathrm{L}$. As to the WHO stage, there were 22 cases of RAEB-1 stage and 18 cases of RAEB-2 stage. As to the IPSS score, there were 0 case of low risk, 23 cases of medium risk, and 17 cases of high risk. As to the karyotype, there were 18 good cases, 14 moderate cases and 8 bad cases. There was no significant difference in age, gender, $\mathrm{WBC}, \mathrm{Hb}, \mathrm{PLT}$, WHO stage, IPSS score and karyotype between the two groups $(P>0.05)$; therefore, the results were comparable. This study was approved by the ethics committee (Ref\# 160, Dated: January 1, 2020) of our hospital.

Inclusive criteria: (1) The diagnosis and classification of MDS patients referred to Expert Consensus on Diagnosis and Treatment of Myelodysplastic Syndrome of Chinese Society of Hematology of Chinese Medical Association and the WHO standard for hematolymph malignancies; ${ }^{8}$ (2) the patients were diagnosed after bone marrow puncture; (3) the patients signed the informed consent.

Exclusive criteria: The exclusive criteria included: (1) transforming into leukemia; (2) having seriously damaged liver and kidney function which was difficult to treat; (3) the survival time shorter than 3 months; (4) failed follow-up observation; (5) having severe infection.

Therapeutic method: F CAG/ DA scheme was used in the traditional group. CAG: aclacinomycin was intravenously injected, $20 \mathrm{mg} /$ time, from the first day to the fifth day; cytarabine was intravenously injected at a dose of $15 \mathrm{mg}$ / time, from the first day to the $14^{\text {th }}$ day, twice each day; recombinant human granulocyte colony-stimulating factor injection was intravenously injected at the dose of $300 \mu \mathrm{g} /$ time, from the first day to the $14^{\text {th }}$ day, twice each day. DA: daunorubicin was intravenously injected at the dose of $60 \mathrm{mg} /$ time, from the first day to the third day, twice each day; cytarabine was intravenously injected at the dose of $100 \mathrm{mg}$ / time, from the first day to the fifth day, twice each day. The patients in the combined group were treated with the scheme of DAC combined with CAG/ DA. The CAG/DA scheme was the same as above, and DAC was given $20 \mathrm{mg}$ each time. Four courses were taken as one course, and the patients in the two groups were treated for four courses.

Supportive treatment and nursing: The patients in the two groups were given symptomatic support treatment such as nutrition support, hydration, alkalization, vomit stopping and stomach care. For example, if $\mathrm{Hb}<60 \mathrm{~g} / \mathrm{L}$, suspension red blood cells were infused; if PLT $<20 \times 10^{9} / \mathrm{L}$ or there was bleeding tendency, platelet was infused; if granulocyte $<0.5 \times 10^{9} / \mathrm{L}$ or granulocytopenia was combined with severe infection, granulocyte colony-stimulating factor was infused; patients with infection was given anti-infection treatment. Moreover, comfort care intervention was also used. The specific contents included: (1) psychological nursing: once MDS patients were diagnosed, they were prone to produce negative emotions such as tension and anxiety; therefore the nursing staff patiently explained the basic knowledge, treatment 
Treatment of myelodysplastic syndrome

Table-I: Chemotherapy effect between two groups [n(\%)].

\begin{tabular}{lccccc}
\hline Group & $C R$ & $P R$ & $H I$ & NR & Total effective rate \\
\hline Traditional group $(\mathrm{n}=40)$ & $10(25.0)$ & $6(15.0)$ & $18(45.0)$ & $6(15.0)$ & $16(40.0)$ \\
Combined group $(\mathrm{n}=40)$ & $19(47.5)$ & $10(25.0)$ & $8(20.0)$ & $3(7.5)$ & $29(72.5)$ \\
$\mathrm{X}^{2}$ & $/$ & $/$ & $/$ & $/$ & 5.971 \\
$\mathrm{P}$ & $/$ & $/$ & $/$ & $/$ & $<0.05$ \\
\hline
\end{tabular}

measures, outcome and prognosis of the disease to the patients and their families and inform them of the possible adverse reactions, precautions and response measures caused by chemotherapy, so as to help the patients to stabilize their emotions; (2) diet nursing: patients were asked to eat foods rich in iron, protein and vitamins, have more meals a day and less food at each, and balance nutrition; (3) life nursing: patients were instructed to work and rest regularly, establish good living habits, exercise moderately, strengthen self-protection awareness, and prevent complications; (4) complication nursing during chemotherapy, nurses closely observed whether patients had anemia, bleeding, infection, etc.

Evaluation of treatment effect: After the treatment, the clinical effect of the two groups was evaluated. According to IWG2006 standard which was revised according to the criteria of therapeutical effect of international working group, the treatment effect was divided into complete remission (CR), partial remission (PR), hematological improvement (HI) and no remission (NR). The total effective rate was calculated by: total effective rate $=(C R+P R) /$ total number of cases $\times 100 \%$. The evaluation of adverse reactions referred to the CTCAE v3.0 standard adopted by the National Cancer Institute (NCI). The end point of the study was death or deadline of follow up. Progression free time (PFS) refers to the time from the beginning of treatment to disease progression or death. Overall survival (OS) refers to the time from the beginning of disease diagnosis to death or deadline of follow up.

Statistical analysis: SPSS 23.0 was used. Mean \pm standard deviation was used for statistical software. Paired-t test was used. The categorical data was compared using Chi-square test. Kaplan-Meier method was used for survival analysis. Difference was considered as statistically significant if $\mathrm{P}<0.05$.

\section{RESULTS}

The total effective rate of the combined group was $72.5 \%$, which was significantly higher than that of the traditional group $(40 \%)(\mathrm{P}<0.05$, Table I). There was no significant difference in adverse reactions between the two groups ( $P>0.05$, Table II). The follow up ended on August 2019. The median follow-up time was 18 months (5-30 months). In the traditional group, the median OS was 18 (530) months, the median PFS was 8 (3-25) months, and 10 patients transformed into acute myeloid leukemia. In the combined group, the median OS was 19 (7-29) months, the median PFS was 11 (226) months, and five patients transformed into acute myeloid leukemia. There was no significant difference in OS between the two groups $\left(X^{2}=0.007\right.$, $\mathrm{P}>0.05)$. Compared with the traditional group, PFS in the combined group was significantly higher, and the difference was statistically significant $\left(\mathrm{X}^{2}=6.862, \mathrm{P}<0.05\right)$.

Among 80 cases of MDS, 45 cases were effective and 35 cases were ineffective. The results showed that the $\mathrm{Hb}$ level, WHO stage and chromosome karyotype of patients before treatment had a significant impact on the treatment effect of MDS, and the difference was statistically significant $(\mathrm{P}<0.05$, Table III).

\section{DISCUSSION}

At present, clinical treatment schemes for MDS include blood transfusion and infusion of PLT, colony stimulating factor, biological response regulator, immunosuppressant, hematopoietic stem cell transplantation, etc., ${ }^{9}$ Although hematopoietic stem cell transplantation is the only

Table-II: Adverse reactions caused by chemotherapy between two groups [ $\mathrm{n}(\%)]$.

\begin{tabular}{lccccc}
\hline Group & WBC reduction & Hb reduction & Infection & Nausea and vomiting & Mucositis \\
\hline Traditional group $(\mathrm{n}=40)$ & $11(27.5)$ & $8(20.0)$ & $10(25.0)$ & $19(47.5)$ & $5(12.5)$ \\
Combined group $(\mathrm{n}=40)$ & $14(35.0)$ & $5(12.5)$ & $8(20.0)$ & $25(62.5)$ & $10(25.0)$ \\
$\mathrm{X}^{2}$ & 0.544 & 0.437 & 0.549 & 1.283 & 1.227 \\
$\mathrm{P}$ & $>0.05$ & $>0.05$ & $>0.05$ & $>0.05$ & $>0.05$ \\
\hline
\end{tabular}


Tangxia Liu et al.

Table-III: QLQ-C30 scale score between two groups (point, Mean \pm SD).

\begin{tabular}{|c|c|c|c|c|c|}
\hline Influence factor & & Effective $(n=45)$ & Ineffective $(n=35)$ & $X^{2}$ & $P$ \\
\hline \multirow[t]{2}{*}{ Age } & $\geq 60$ & $11(24.4)$ & 13(37.1) & 0.749 & $>0.05$ \\
\hline & $<60$ & $34(75.6)$ & $22(62.9)$ & & \\
\hline \multirow[t]{2}{*}{ Gender } & male & $31(68.9)$ & $20(57.1)$ & 0.407 & $>0.05$ \\
\hline & female & $14(31.1)$ & $15(42.9)$ & & \\
\hline \multirow[t]{2}{*}{ WBC } & $\geq 4(\times 109 / \mathrm{L})$ & $34(75.6)$ & $24(68.6)$ & 0.281 & $>0.05$ \\
\hline & $<4(\times 109 / \mathrm{L})$ & $11(24.4)$ & $11(31.4)$ & & \\
\hline \multirow[t]{2}{*}{ PLT } & $\geq 100(\times 109 / \mathrm{L})$ & 14(31.1) & $4(11.4)$ & 3.802 & $>0.05$ \\
\hline & $<100(\times 109 / \mathrm{L})$ & $31(68.9)$ & $31(88.6)$ & & \\
\hline \multirow[t]{2}{*}{$\mathrm{Hb}$} & $\geq 90 \mathrm{~g} / \mathrm{L}$ & $16(35.6)$ & $3(8.6)$ & 4.779 & $<0.05$ \\
\hline & $<90 \mathrm{~g} / \mathrm{L}$ & $29(64.4)$ & 32(91.4) & & \\
\hline \multirow[t]{2}{*}{ WHO staging } & RAEB-1 & $19(42.2)$ & $27(77.1)$ & 6.011 & $<0.05$ \\
\hline & RAEB-2 & $26(57.8)$ & $8(22.9)$ & & \\
\hline \multirow[t]{2}{*}{ IPSS score } & Low risk+medium risk & $29(64.4)$ & $20(57.1)$ & 0.488 & $>0.05$ \\
\hline & High risk & $16(35.6)$ & $15(42.9)$ & & \\
\hline Chromosome & Good+moderate & $42(93.3)$ & $24(68.6)$ & 5.027 & $<0.05$ \\
\hline karyotype & Bad & $3(6.7)$ & $11(31.4)$ & & \\
\hline
\end{tabular}

promising method to cure at present, MDS patients who are mostly elderly and often have a variety of basic diseases cannot tolerate hematopoietic stem cell transplantation. ${ }^{11}$ Although the traditional chemotherapy scheme can achieve short-term remission effect, the effect lasts for a short time, and high-intensity treatment will cause chemotherapyrelated complications, increase mortality and result in poor prognosis. ${ }^{12}$ It has been found that DNA abnormal methylation plays an important role in the occurrence and progress of MDS. ${ }^{13}$ DNA methylation is an important epigenetic modification. Abnormal DNA methylation can lead to the silencing of tumor suppressor genes and the abnormal regulation of normal cell growth and differentiation, and the damaged DNA cannot be repaired in time. ${ }^{14,15}$

DAC is a kind of adenosine analogue of deoxycytidine acid, which belongs to methyltransferase inhibitor. It can reduce DNA methylation by inhibiting DNA methyltransferase, make the tumor suppressor gene return to normal, reactivate the gene inactivated due to DNA excessive methylation, and make the process of cell differentiation, aging or apoptosis return to normal. ${ }^{16,17} \mathrm{~A}$ study of Ye et al. enrolled 81 cases of MDS-RAEB, ${ }^{18} 40$ patients were treated with decitabine before chemotherapy, and the median follow-up time was 10.9 months. Through comparing the results of chemotherapy alone with that of DAC before chemotherapy, it was found that the objective response rate (ORR) and CR rate of the decitabine group was significantly higher than that of chemotherapy group. It further suggested that the effect of DAC in treating MDS was significant. In this study, the total effective rate of patients treated with DAC combined with CAG/DA was higher than that of patients treated only with CAG/DA, which was consistent with the previous reports. However, the ORR and CR rate of this study were lower than those of Jeong et al.. ${ }^{19}$ The reasons are as follows. This study included more highrisk and extremely high-risk patients; thus the risk degree was higher. Moreover, the medium treatment course of patients with effective DAC treatment result was eight in the study of Jeong et al. and that of patients who received decitabine treatment in this study was four. Some patients stopped treatment because of economic reasons, compliance or severe complication in the process of medicine use, and DAC has not taken effect at that time, leading to reduced effective rate.

The analysis of the survival condition between the two groups suggested that the difference of OS between the two groups had no statistical significance and the combined group had significantly higher PFS than the traditional group, which suggested that decitabine could increase the disease-free survival time of MDS patients. Wen et al. found that the median OS of high-risk MDS patients who were treated by decitabine was significantly higher than that of chemotherapy. ${ }^{20}$ The difference between the result of Wen et al. and this study might be that the sample size was small and the follow up time was short. 
In addition, this study also innovatively analyzed factors that affected the treatment effect of MDS patients. It was found that the $\mathrm{Hb}$ level, WHO stage and chromosome karyotype of patients before treatment had a significant impact on the treatment effect of MDS and the treatment effect of patients with $\mathrm{Hb}<90 \mathrm{~g} / \mathrm{L}$, RAEB-1 stage and bad chromosome karyotype was often poor.

\section{CONCLUSION}

In conclusion, the effect of DAC combined with conventional chemotherapy in the treatment of MDS is good, which will not increase the adverse reactions of patients. In addition to the treatment scheme, the influence factors of effect of MDS also include $\mathrm{Hb}$, WHO stage and chromosome karyotype of patients.

Declaration of interest: All authors declared there was no conflict interests involved.

\section{Grant Support \& Financial Disclosures: None.}

\section{REFERENCES}

1. Paridar M, Ghalesardi OK, Seghatoleslami M, Ahmadzadeh A, Khosravi A, Saki N. Cytogenetic and molecular basis of BCRABL myelodysplastic syndrome: Diagnosis and prognostic approach. J Cancer Metast Treatment. 2017;3(2):38-44. doi: 10.20517/2394-4722.2016.61

2. Michel G, Galambrun C, Sirvent A, Pochon C, Bruno B, Jubert $\mathrm{C}$, et al. Single-vs double-unit cord blood transplantation for children and young adults with acute leukemia or myelodysplastic syndrome. Blood. 2016;127(26):3450-3457. doi: 10.1182/blood-2016-01-694349

3. Zhuang L, Ma Y, Wang Q, Xu XP, Zhang J, Zhu C, et al. Establishment of a myelodysplastic syndrome transformed to leukemia cell line stably expressing green fluorescent protein. J Exp Hematol. 2017;1487-1492. doi: 10.7534/j.issn.10092137.2017.05.036

4. Pang YB, Fan LX, Xue H, Liu J, Du X, Hua LM, et al. Mechanism and its countermeasure of hypomethylating agent resistance in patients with myelodysplastic syndrome-review. J Exp Hematol. 2018;11(5):1574-1578. doi: 10.7534/j.issn.10092137.2018.05.053

5. Saunthararajah Y, Sekeres M, Advani A, Mahfouz R, Durkin L, Radivoyevitch T, et al. Evaluation of noncytotoxic DNMT1depleting therapy in patients with myelodysplastic syndromes. J Clin Invest. 2015;125(3):1043-1055. doi: 10.1172/JCI78789

6. Ma YY, Zhao M, Liu Y, Zhao DF, Wang LX, Chen XP, et al Use of decitabine for patients with refractory or relapsed acute myeloid leukemia: A systematic review and meta-analysis. Hematol (Amsterdam, Netherlands). 2019;24(1):507-515. doi: $10.1080 / 16078454.2019 .1632407$

7. Klco JM, Spencer DH, Lamprecht TL, Sarkaria SM, Wylie $\mathrm{T}$, Magrini $\mathrm{V}$, et al. Genomic impact of transient lowdose decitabine treatment on primary AML cells. Blood. 2013;121(9):1633-1643. doi: 10.1182/blood-2012-09-459313

8. Chinese Society of Hematology, Chinese Medical Association. Expertconsensus on diagnosis and treatment of myelodysplastic syndrome (2014). Chin J Hematol. 2014;35(11):1042-1048. doi 10.3760/cma.j.issn.0253-2727.2014.11.023
9. Suh KJ, Cheong JW, Kim I, Kim HJ, Shin DY, Koh Y, et al. Prognostic impact of IPSS-R and chromosomal translations in 751 korean patients with primary myelodysplastic syndrome. PLoS One. 2016;11(11): e0166245.

10. Lindsley RC. Uncoding the genetic heterogeneity of myelodysplastic syndrome. Hematol Am Soc Hematol Educ Program. 2017;2017(1):447-452. doi: 10.1182/ asheducation-2017.1.447

11. Yan XF, Wei JY, Wang JH, Ren YL, Zhou XP, Mei C, et al. Analysis of the karyotype abnormalities and its prognostic in 298 patients with myelodysplastic syndrome. Chin J Hematol. 2015;36(4):297-301. doi: 10.3760/cma.j.is sn.0253-2727.2015.04.008

12. Fu HY, Zhou HR, Yan JG, Chen CJ, Shen JZ. Clinical significance of hypermethylation of DLC-1 gene in myelodysplastic syndrome patients and effects of decitabine on DLC-1 gene expression. Nat Med J China. 2017;97(6):412-417. doi: 10.3760/ cma.j.issn.0376-2491.2017.06.003

13. Li JY, Chen YY, Zhu Y, Zhou JF, Xu YL, Li Y, et al. Efficacy and safety of decitabine in combination with G-CSF, lowdose cytarabine and aclarubicin in newly diagnosed elderly patients with acute myeloid leu-kemia. Oncotarget. 2015;6(8):6448-6458.

14. Zhang YP, Wu WZ, Cui GX. Comparison of clinical efficacy between decitabine combined with CAG regimen and CAG regimen alone in patients with intermediate to high-risk myelodysplastic syndromes. J Exp Hematol. 2014;22(5):13411344. doi: 10.7534 /j.issn.1009-2137.2014.05.030

15. Carella AM. Clinical outcome after failure of hypomethylating therapy for myelodysplastic syndromes. Eur J Haematol. 2015;94(6):463. doi: 10.1111/ejh.12521

16. Ye XN, Zhou XP, Wei JY, Xu GX, Li Y, Mao LP, et al. Epigenetic priming with decitabine followed by low-dose idarubicin/cytarabine has an in-creased anti-leukemic effect compared to traditional chemotherapy in high-risk myeloid neoplasms. Leiik Lymphoma. 2016;57(6):1311. doi: 10.3109/10428194.2015.1091931

17. Meldi K, Qin T, Buchi F, Droin N, Sotzen J, Micol JB, et al. Specific molecular signatures predict decitabine response in chronic myelomonocytic leukemia. J Clin Invest. 2015;125(5):1857-1872. doi: 10.1172/JCI78752

18. Ye L, Ren YL, Zhou XP, Mei C, Ma LY, Ye XN, et al Decitabine priming prior to low-dose chemotherapy improves patient outcomes in myelodysplastic syndromesRAEB; A retrospective analysis vs. Chemotherapy alone. J Cancer Res Clin Oncol. 2017;143(5):873-882. doi: 10.1007/ s00432-016-2331-0

19. Jeong SH, Kim YJ, Lee JH, Kim YK, Kin SJ, Park SK, et al. A prospective, muhieenter, observational study of longterm decitabine treatment in patients with myelodysplastic syndrome. Oneotarget. 2015;6(42):44985-44994. doi: 10.18632/ oncotarget.6242

20. Wen XM, Lin J, Yang J, Yao DM, Deng ZQ, Tang CY, et a1. Double CEBPA mutations are prognostically favorable in non-M3 acute myeloid leukemia patients with wild-type NPMl and FLT3.ITD. Int J Clin Exp Pathol. 2014;7(10):6832-6840.

\section{Authors' Contribution:}

TXL: Study design, data collection and analysis. JW \& CML: Manuscript preparation, drafting and revising.

TXL \& LZJ: Review, final approval of manuscript and are responsible for integrity of study. 